\title{
Quantitative Performance Analysis of Flexible Ink-jet Printed Low-cost DNA Sensors Based on a CNT Network
}

This paper was downloaded from TechRxiv (https://www.techrxiv.org).

\section{LICENSE}

CC BY 4.0

SUBMISSION DATE / POSTED DATE

$12-09-2021 / 16-09-2021$

\section{CITATION}

Cai, Liang; Kovur, Krishna M; Kovur, Prashanthi; D. Montemagno, Carlo (2021): Quantitative Performance Analysis of Flexible Ink-jet Printed Low-cost DNA Sensors Based on a CNT Network. TechRxiv. Preprint. https://doi.org/10.36227/techrxiv.16608685.v1

$\mathrm{DOI}$

10.36227/techrxiv.16608685.v1 


\title{
Quantitative Performance Analysis of Flexible Ink-jet Printed Low-cost DNA Sensors Based on a CNT Network
}

\author{
Liang Cai, K. Krishna Mohan, K. Prashanthi*, C. D. Montemagno
}

\begin{abstract}
We report the design, fabrication and quantitative performance analysis of a low-cost, flexible carbon nanotube (CNT) network-based deoxyribonucleic acid (DNA) sensor. These sensors comprise an array of ink-jet printed silver (Ag) electrodes on a transparent polyethylene terephthalate (PET) flexible substrate, where a CNT network acts as a sensing layer. The DNA
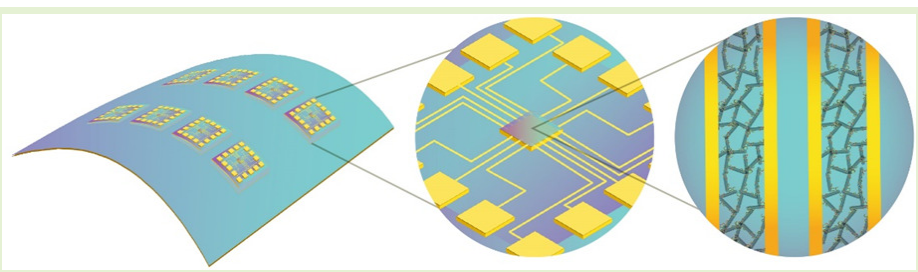

hybridization is studied by immobilizing single-stranded DNA (sSDNA) probes on the CNT surface; these probes recognize their complementary DNA target. Further, we have carried out a quantitative performance analysis of the flexible CNT biosensors using the analytic hierarchy process (AHP). We have identified various influencing factors and sub-factors (performance indicators), and quantified the performance of the flexible CNT biosensors in different measured states (before bending, during bending and after bending). Additionally, the noise and other external factors contributing to the measured real signal have been quantified. The interpretation of the overall outcome will enable the improvement of the performance of flexible biosensors fabricated through large-scale manufacturing for possible commercialization.
\end{abstract}

Index Terms-Analytical hierarchy process (AHP), Carbon Nanotubes (CNTs); DNA sensors; Flexible sensors; Ink-jet Printing; Performance Analysis

\section{INTRODUCTION}

$\mathrm{T}$ HE fabrication of miniaturized, low-cost, flexible sensors based on nanostructure network array is expected to provide important benefit in physical, chemical and biological detection[1]-[7], enabling applications such as personal health and environmental sensing[4]-[6], [8], [9]. In this regard, carbon-based nanomaterials such as carbon nanotubes (CNTs) and graphene have been extensively investigated as response components in flexible biosensors due to their unique electrical and mechanical properties[10][14]. In particular, CNTs are a stiff and light material that exhibits a high Young's modulus and good electrical conductivity without electron migration issues[2]. As a consequence of these remarkable properties, CNTs have become a promising candidates for a wide range of applications [3], [7], [15], [12] [13][2], [16], [17].

This paragraph of the first footnote will contain the date on which you submitted your paper for review. It will also contain support information, including sponsor and financial support acknowledgment. This work was supported by Government of Alberta and Alberta Innovates Technology Futures.

Liang Cai is with the Department of Electrical \& Computers Engineering, University of Alberta, Edmonton, T6G 2V4, Canada (email: Icai1@ualberta.ca). K. Krishna Mohan, and K. Prashanthi are with the Department of Chemical and Materials Engineering, University of Alberta, Edmonton, T6G 2V4, Canada (e-mail: kkmiitb@gmail.com, kovur@ualberta.ca,).

Note: Dr. C.D. Montemagno was died on 11th October 2018.
The development of CNT biosensors that transduce a biological or chemical stimulus into an electrical signal has high potential for miniaturization and integration. Moreover, label-free electronic methods promise to offer sensitivity, selectivity, and low cost for the detection of biological species. The most important component of electrical signal-based CNT biosensors is the transduction mechanism from CNTs which is responsible for converting the responses of bio-analyte interactions into a detectable electrical signal in a reproducible manner. Efficient bulk fabrication of nanobiosensor devices requires either bottom-up self-assembly or drop casting approaches for modifying electrodes with nanomaterials. Drop casting is one of the most commonly used methods in low-cost biosensor fabrication as it is a rapid method and does not require complicated apparatuses. Since, drop casting is a stochastic process, it is hard to control the repeatability and uniformity, and therefore, the overall performance of the fabricated devices. Further, the performance of electronics on flexible substrates is known to degrade due to bending stress leading to reduced device performance. When manufacturing large-scale flexible nanobiosensors for possible commercialization, the validation of parameters, such as the long-term stability of the entire device and the effects of repeated bending stress is essential. Therefore, it is important that we address the factors affecting this electrical signal variation in fabricated flexible devices in order to improve their performance. To compare the relative influence of varying factors on device function and reliability, a 
performance evaluation system may be utilized. The analytical hierarchy process (AHP) methodology developed by Saaty [18]-[20] delivers a way to address complex decisions containing several attributes of analyzing risks [21]. This technique has been widely adopted by several researchers in the context of health-care [22]-[24], wireless sensor networks [25] and biosensors [26], [27]. However, until now, the performance analysis of flexible sensors has been accomplished experimentally based on qualitative evaluation but not quantitatively [28]-[30]. Recently, we have reported the risk assessment of multiplexed CNT network based DNA sensors fabricated on silicon substrates using AHP [26]. Here, we report the design, fabrication of CNT network- based DNA sensors on a flexible substrate by combining ink-jet printing and drop casting. We have conducted quantitative performance analysis for flexible CNT DNA sensors at various measured states (before bending, during bending and after bending) using AHP. We have further quantified the noise and other external factors contributing to the real signal during sensing. Hence, in the present work, the AHP based quantitative model has been applied to a flexible biosensor for the first time to evaluate its performance analysis under various measured states.

\section{EXPERIMENTAL METHODS}

Probe, target and control single-stranded DNA (ssDNA) were purchased from IDT (USA). Each strand consisted of 30 bases, with sequence length $\sim 10.2 \mathrm{~nm}$. The probe ssDNA had the following sequence: 5'-amino-C6-TGA CTT GAG ACC AGT TGA ATA AAA AAA AAA-Cy3-3' (MW 9288 g/mol). The complementary ssDNA had the following sequence: 5'amino-C6-TTT TTT TTT TAT TCA ACT GGT CTC AAG TCA-3' (MW $9297 \mathrm{~g} / \mathrm{mol}$ ). The control ssDNA had the following sequence: 5'-phosphate-TTT TTT TTT TTG ACT TGA GAC CAG TTG AAT-3' (MW 9278 g/mol).

The sensor electrodes (width $100 \mu \mathrm{m}$ ) were directly printed on a polyethylene terephthalate (PET) sheet as well as selfadhesive vinyl paper using a prototype ink-kit made of conductive silver (Ag) nanoparticles (AgIC, Japan). This printable silver ink is compatible with many home inkjet printers and quickly dries and becomes conductive without a need for sintering. Single-walled carbon nanotubes (SWCNTs) with an average diameter of $0.83 \mathrm{~nm}$ and a median length of 1 $\mu \mathrm{m}$ (Sigma -Aldrich, product\#704121) were functionalized with probe ssDNA by adsorption as previously reported [31]. The prepared DNA-SWCNT solutions (with concentration of $1 \mu \mathrm{g} / \mathrm{ml}$ ) were dropped onto the sensor electrodes using a pipette $(0.5-10 \mu \mathrm{l})$, forming circles with diameters of approximately $1 \mathrm{~mm}$. Images of ssDNA-SWNT samples were acquired using atomic force microscopy (AFM) (Dimension Edge) in the tapping mode in air. A Keithley 6220 DC Precision Current Source and a Keithley 2182A Digital Nanovoltmeter were used to measure the electrical characteristics of flexible CNT network biosensors. LabVIEW, an automated data acquisition software was used to collect the data. The electrical sensing of DNA hybridization on ssDNA functionalized CNT network array was carried out in response to the addition of complementary (target) and

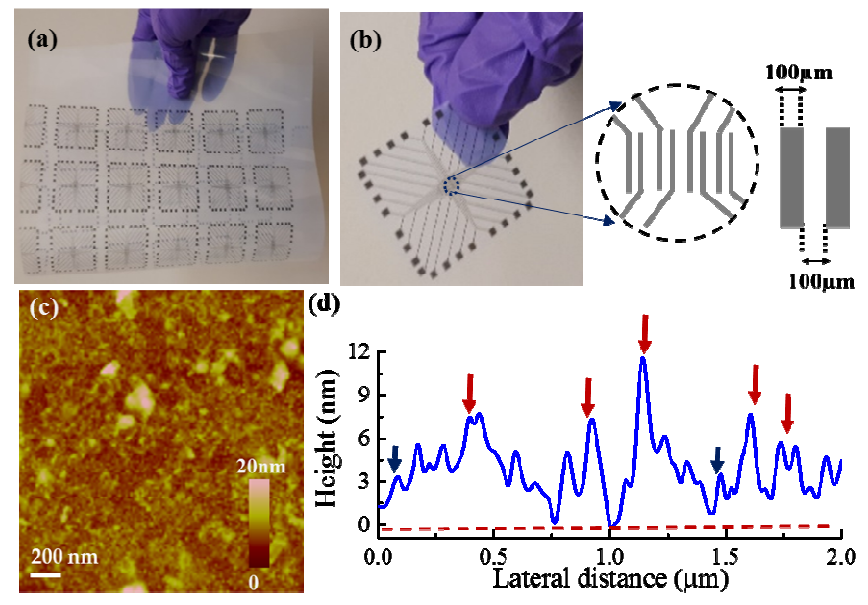

Fig. 1 (a) Fabricated array of Ag electrodes on flexible PET sheet (b) photographic image of single device with functionalized CNT network on Ag electrode array. Zoomed portion shows the illustration of CNT network on $\mathrm{Ag}$ electrodes (c) AFM image of DNA origami functionalized CNTs and (d) corresponding height profile shows that CNTs are functionalized with ssDNA.

mismatched (control) oligos in phosphate-buffered saline (PBS, pH 7.4). All experiments were performed at room temperature.

\section{RESULTS AND DISCUSSIONS}

\section{A. Fabrication of CNT devices}

Inkjet-printing is a very promising technique for the deposition of conductive layers. It provides major benefits over screen-printing, due to the adaptability, the high precision, and resolution of the printed patterns[32], [33]. Most importantly, this technique can be used to deposit small volumes ( picolitres) of ink in a quick and low-cost way. An array of the Ag electrodes was directly printed on flexible PET sheets using an ink-jet printer. The prepared ssDNA functionalized CNT solution was drop cast onto these ink-jet printed electrode arrays. As the solvent evaporates the CNT network is formed; this network adheres to the surface of the PET substrate due to Van der Waals forces[10], [34]. Normally, the conductance of the CNT network is induced by the conducting paths between CNTs and the electrodes. Schematics and photographic images of CNT network array sensors on flexible substrates are shown in Fig. 1(a) and (b), respectively. After printing, the PET sheet was then cut into individual devices as shown in Fig. 1(c). The enlarged portion of Fig. 1(c) represents the schematic of ssDNA functionalized CNT network on printed Ag electrodes. In the design, each big square represents one device. Each device has 16 arrays with 2 electrodes per array (total 32 electrodes per device). Here, we have measure signal between two electrodes of each array. Fig. 1(d) shows the AFM topography image while Fig. 1(e) shows the corresponding height profile of DNA functionalized CNTs. The AFM topography image showed a bead-string-like structure of SWNTs, indicating the wrapping of ssDNA along SWNT sidewalls. As indicated by the crosssectional AFM topography height profile, the height of bare 
CNT from the substrate is about 1-3 $\mathrm{nm}$ (blue arrows). However, after ssDNA adsorption, the height of CNT becomes $6-12 \mathrm{~nm}$ (red arrows). This measured height confirms the functionalization of DNA onto the CNT surface.

\section{B. Electrical resistance measurements}

Fig. 2(a) presents measured resistance variations from ink-jet printed Ag electrodes on the flexible PET substrate; the average resistance value was $11 \pm 2 \Omega$. This low variation in resistance reflects the uniformity and repeatability of the inkjet printing process. Fig. 2(b) shows the normalized resistance (the ratio of resistance measured during bending to the initial resistance) of the electrodes after during 0 to 120 bending cycles. It is observed that resistance increases with the increasing number of bending cycles, however, the resistance increased by only a factor of around two over 120 bending cycles. Such a low resistance change reflects the remarkable robustness of the sensors to mechanical deformations, and is associated with the stress-enduring $\mathrm{Ag}$ ink formulations used for the sensor printing.
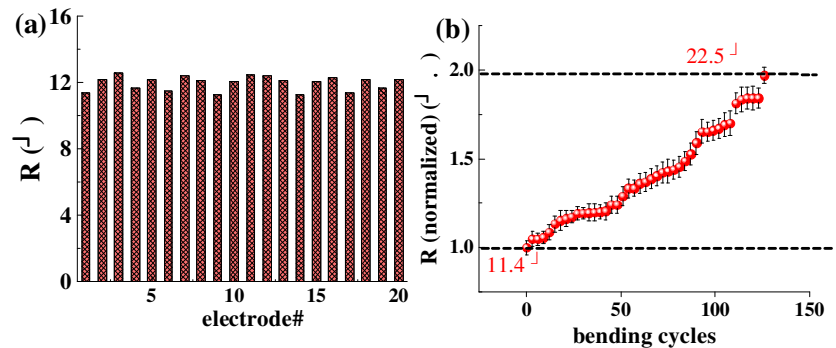

Fig. 2. (a) Measured resistance variations from ink-jet printed silver electrodes of same device (b) Normalized resistance of ink-jet printed silver electrodes as a function of bending cycles.

Fig. 3 (a) shows experimentally measured real-time resistance of three flexible ssDNA functionalized CNT biosensor devices on the same PET substrate. For each device, the resistance of at least five different ssDNA functionalized CNT arrays was measured. As can be seen in Fig. 3, the resistance of the ssDNA functionalized CNTs ranged from $140 \mathrm{k} \Omega$ to $148 \mathrm{k} \Omega$, and fluctuated over time. The average resistance values varied from device to device, as well as between the arrays of a single device. Fig. 3(b) summarizes the measured resistance of five functionalized CNT network arrays comprising three devices.

Fig. 4 illustrates the characterization of the devices in response to mechanical deformation. Fig. 4 (a) shows the dynamic resistance variation from the CNT biosensor during consecutive iterations of mechanical bending and relaxed states. The resistance varies by around $10 \%$ during this process, increasing during bending and decreasing upon relaxation. The schematic representation of various measured states; before bending (C1), during bending (C2) and after bending (C3) of flexible sensors is shown in Fig. 4(b). The devices were consistently bent to introduce a tensile stress to the active layer, as this is expected to result in a higher response than a compressive stress due to the fact that current is carried through an interconnected network. Fig. 4(c)
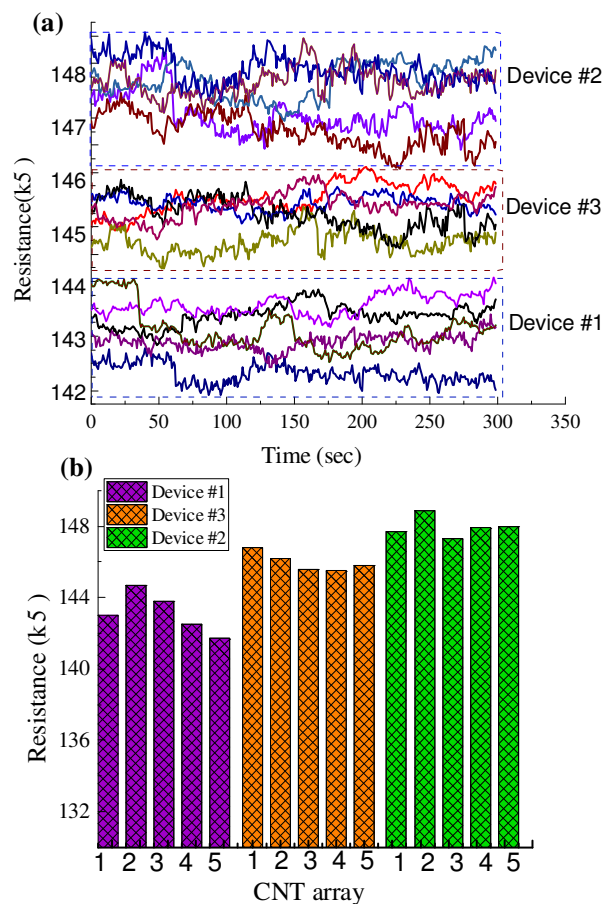

Fig. 3. (a) Measured real-time signal variation from different arrays of flexible ssDNA functionalized CNT for three devices (b) Resistance measured for three devices consisting of ssDNA functionalized CNT sensors.
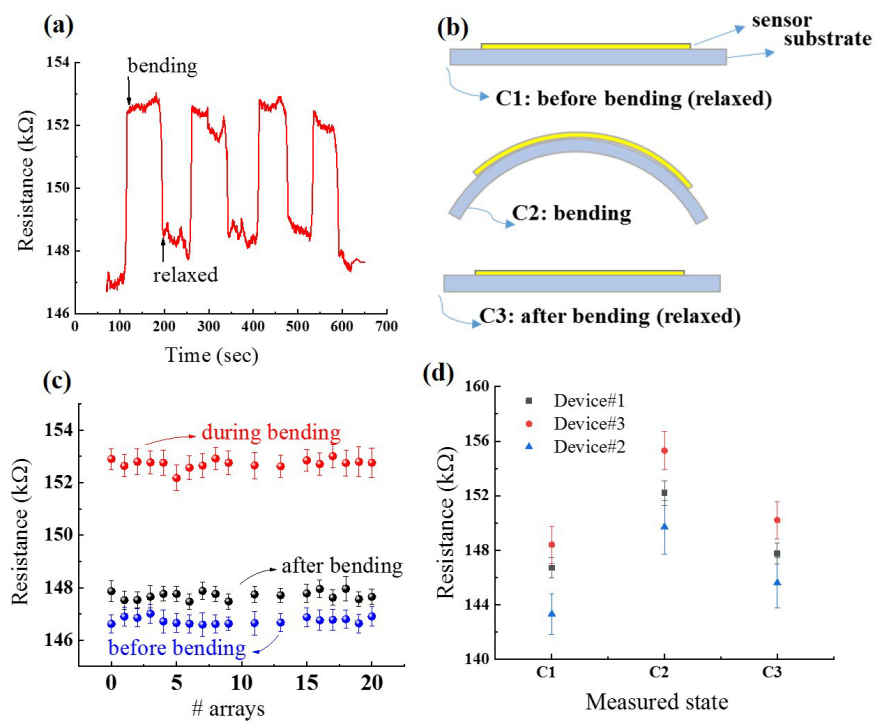

Fig. 4. Dynamic resistance variation from the CNT sensor during consecutive iterations from complete bending of the sensor. (b) Schematic showing relaxed and bending modes of the flexible sensor (c) Measured resistance as a function of measured arrays of CNT sensor before bending (C1), bending (C2) and after bending (C3). (d) Resistance plotted against 3 measured states $(\mathrm{C} 1, \mathrm{C} 2, \mathrm{C} 3)$ for three $\mathrm{CNT}$ devices. The error bars indicate the variability of measured data for different arrays of the device.

represents resistance as a function of measured cycles of CNT biosensor for three states $(\mathrm{C} 1, \mathrm{C} 2, \& \mathrm{C} 3)$. The resistance plotted against three measured states $(\mathrm{C} 1, \mathrm{C} 2, \& \mathrm{C} 3)$ for three CNT biosensor devices is summarized as Fig. 4(d). The error bars indicate the variability of measured data for different arrays of the same device. It was observed that resistance of the device increases during bending. Since the CNT network is formed by a large number of randomly arranged individual 

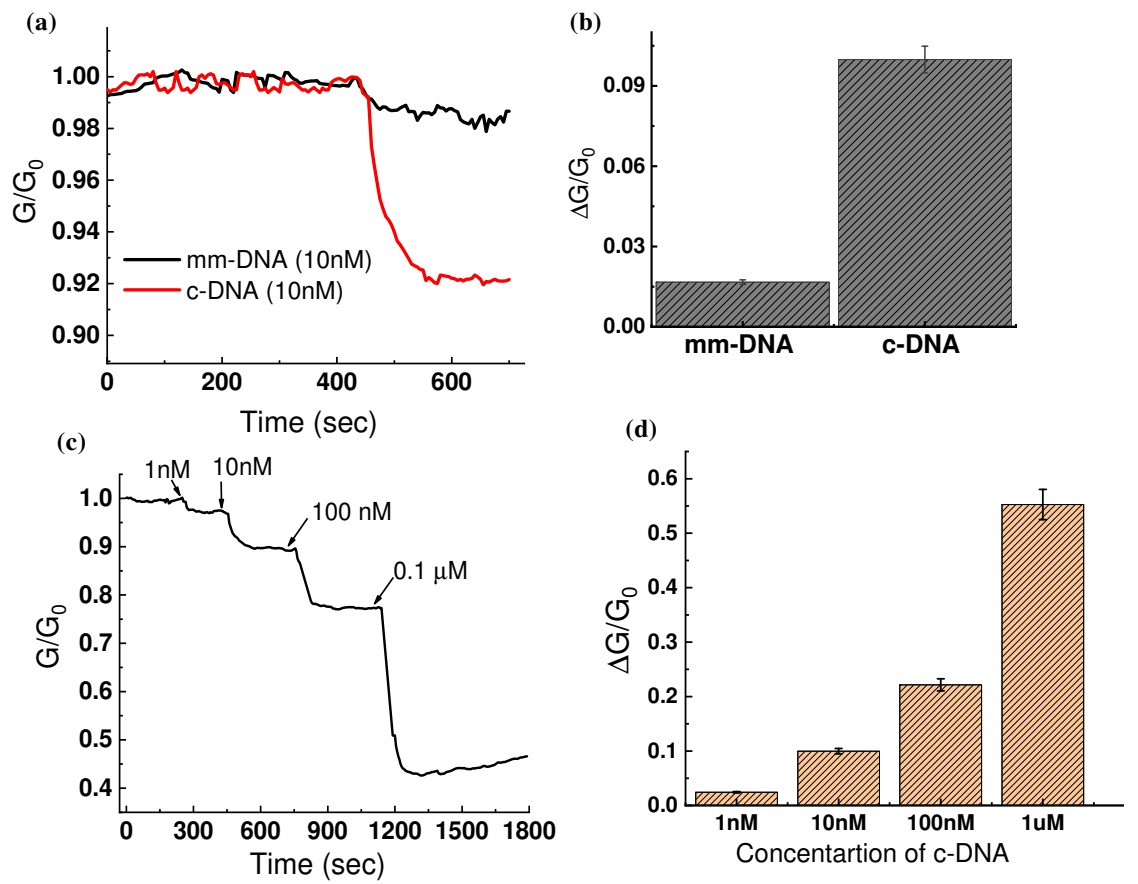

Fig. 5. (a) Response of ssDNA functionalized CNT network array towards complementary (c-DNA) and control/mismatch DNA (mm-DNA). (b) Bar diagram showing the signal of a CNT device functionalized with ssDNA exhibits selective response to the addition of complementary DNA (c-DNA). (c) Real-time monitoring of DNA hybridization on CNT network biosensor at various c-DNA concentrations. (d) Bar diagram showing signal shift of CNTs with increasing DNA concentration (The error bars represent the standard deviations of 3 parallel samples at each target or complementary DNA (c-DNA)

CNTs, two types of resistances determine the total resistance of the sensor[10], [35]. The first type is the intrinsic resistance of the CNTs and the second type is junction resistance between connected CNTs[10], [35]. Here the shift in the resistance may be a result of the combined effect of intrinsic (piezoresistive) and junction resistance of the CNT network due to bending stress. The observed piezoresistivity in CNT network can be mainly attributed to the significant variation of conductive networks formed by CNTs; tunneling resistance change in neighboring CNTs due to distance change; piezoresistivity of CNTs themselves due to their deformation[11]. As observed in Fig. 4(a), the sensor response during bending to relaxation cycle is unstable because of the alteration of connectivity or conductive path between CNTs in the network. We believe that ink-jet printing of CNTs directly on electrodes may improve the mechanical stability of CNT network biosensors.

\section{DNA hybridization test}

Specificity of ssDNA functionalized CNTs towards complementary DNA (c-DNA) was measured against control or a mismatched DNA (mm-DNA) in buffer at a concentration of $10 \mathrm{nM}$ (Fig. 5 (a)). The capture probe ssDNA serves as a platform for binding with complementary DNA in the process of hybridization. A larger conductance change was observed with complementary DNA over mismatched DNA, indicating the specificity of the functionalized CNT network array sensor towards c-DNA as shown in a bar diagram (Fig. 5(b)). Fig. $5(\mathrm{c})$ presents the DNA sensitivity test with the concentrations of c-DNA $(1 \mathrm{nM}-0.1 \mu \mathrm{M})$. The electrical measurements demonstrated a linear relationship between the change in voltage and concentrations of c-DNA. Fig. 5(d) presents a bar diagram of the response of CNT biosensor with increasing
DNA concentration (The error bars represent the standard deviations of 3 independent sensors at each target concentration). The sensing mechanism of CNTs results from different sources; the change in the electrical conductance can be attributed to electron doping by DNA hybridization on the CNT sidewall [31], modulation of the contact resistance [36], variation in the Schottky barrier [37] as a result of DNA charge transfer, and conductance modulation as a result of contact metal work function change due to the adsorbed species[38], [39]. Nevertheless, the results indicate that the proposed label-free detection technique for DNA hybridization with high sensitivity and selectivity is possible by direct electrical detection on functionalized CNT network. The flexible substrates used for fabricating functionalized CNT network devices shall give more leverage in realizing portable and disposable DNA sensors with applications in molecular biology, point-of-care medical diagnostics, pathological investigations, genotyping, etc.

\section{PERFORMANCE ANALYSIS}

In this section, we present quantitative analysis using AHP to assess the performance of flexible CNT biosensor in three measured states categorized as Case 1: before bending (C1); Case 2: During bending (C2); Case 3: After bending (C3). After a careful study of relevant parameters, we have identified two factors and a number of corresponding subfactors that lead to variation in device performance. The first factor is Junction resistance (F1), and associated sub-factors include metal CNT band alignment (SF1); contact area change (SF2) and change in work function (SF3). The second factor is Intrinsic piezoresistance (F2), and related sub-factors are variation of conductive resistance formed by CNT network (SF4); tunneling resistance change in neighboring CNTs 
(SF5); and self piezoresistance of CNTs (SF6). Additionally, we have quantified the noise and other external factors contributing to the real signal of the CNT biosensor through several experimental trials. The quantitative performance analysis of flexible CNT biosensors has been done via:

- Classifying multiple influencing factors (factors, subfactors) of conflicting nature and structuring the application problem observed during experimentation of flexible CNT biosensors

- Computing influencing factors via. relative importance and likeliness impact to evaluate the priorities across the application problem

- Quantifying the overall outcome on the basis of the computed factors with regard to:

$\checkmark$ influencing factors -during the three measured states of $C 1, C 2$, and $C 3$ of flexible CNT biosensors

$\checkmark$ signal - the contribution of noise and external factors to real signal

- Interpreting the overall outcome through the quantified results to improve the performance of flexible CNT sensors

\section{A. AHP procedure}

Here, we provide a step by step description in the AHP procedure

Step 1: Define problem formulation from the objective's perspective to assess the performance analysis analysis (e.g. how is the performance of flexible CNT sensors affected by various factors?)

Step 2: Identify performance influencing factors across hierarchy levels with options and possible outcomes at the lowest level and then establish hierarchical modeling accordingly

Step 3: Establish performance pair-wise comparisons matrices of size $\mathrm{m} \times \mathrm{m}$ across application hierarchy at different levels of the abstractions among the (i) factors; (ii) sub-factors; and (iii) possible options and outcome to carry out performance assessment. For a $\mathrm{m} \times \mathrm{m}$ matrix, we perform $\mathrm{m} *(\mathrm{~m}-1) / 2$ pairwise comparisons. Eq. (1) represents an $(\mathrm{m} \times \mathrm{m})$ matrix shown below:

$\mathrm{Z}=\left[\mathrm{z}_{\mathrm{ij}}\right]=\left[\begin{array}{ccc}1 & \cdots & z_{1 m} \\ \vdots & \ddots & \vdots \\ z_{m 1} & \cdots & 1\end{array}\right]$ where $\mathrm{z}_{\mathrm{ij}}=\frac{1}{z_{j i}}(1)$

for all $\mathrm{i}, \mathrm{j}=1,2, \ldots, \mathrm{m}$

$\mathrm{z}_{\mathrm{ii}}=1$, for all $\mathrm{i}=1,2, \ldots, \mathrm{m}$

If $\mathrm{z}_{\mathrm{ij}}=\alpha \neq 0$, then $\mathrm{z}_{\mathrm{ji}}=1 / \alpha$, for all $\mathrm{i}=1,2 \ldots, \mathrm{m}$

Step 4: Compare relative importance between factors and subfactors and as well as likeliness impact of possible options and outcome through the 9-point scale ratings proposed by Saaty [20]. The scale of relative importance (describing the preference of $z_{i}$ with respect to $z_{j}$ ) is defined as follows in the choice of 1 to 9 . If $z_{i}$ is determined to be extremely important over $\mathrm{z}_{\mathrm{j}}$, the matrix entry is assigned a value of 8 or 9 , and very strongly important for 6 or 7 . Similarly, if $\mathrm{zi}_{\mathrm{i}}$ is strongly important over $\mathrm{Zj}_{\mathrm{j}}$, the entry is assigned a value of 4 or 5 . Finally, 2 or 3 is considered when $\mathrm{zi}$ is moderately important against $\mathrm{zj}$. Hence, for every pair-wise matrix $(\mathrm{m} \times \mathrm{m})$ dimension, we perform $m(m-1) / 2$ judgment entries into the upper triangle matrix $Z_{\mathrm{ij}}$. The reciprocal values of 1 to 9 are inserted into lower triangular pair-wise entries from the upper triangular and finally, the diagonal of matrix entries with a value of 1 .

Thus, mathematically, we denote pair-wise for the matrix $\mathrm{Z}$ as $\mathrm{Z}=\left[\mathrm{z}_{\mathrm{ij}}\right]$; where $\mathrm{z}_{\mathrm{ii}}=1$; and $\mathrm{z}_{\mathrm{ij}}=1 / \mathrm{z}_{\mathrm{ji}}$ as shown in the Eq. (2) $\mathrm{Z}=\left\{\begin{aligned} \mathrm{z}_{\mathrm{ij}}=\frac{1}{\mathrm{z}_{\mathrm{ji}}} ; & \mathrm{i} \neq \mathrm{j} \\ 1 ; & \mathrm{i}=\mathrm{j}\end{aligned}\right.$

Step 5: Assessment of pair-wise matrix:

Here, in this step 4, we compute vector of weights characterized by principal eigenvector from its corresponding maximum eigenvalue $\lambda_{\max }$ of $\mathrm{Z}$ for all judgement matrices formulated in the step 4 followed by consistency check. Therefore, we obtain a normalized eigenvector to evaluate the weights in the AHP that measures the consistency of vector of preferences with respect to outcome of a specific matrix. Thus, we represent matrix $\mathrm{Z}$, its eigenvalue and normalized eigenvector, denoted mathematically as $\mathrm{ZW}=\lambda_{\max } \mathrm{W}$, or $\mathrm{ZW}=\left(\lambda_{\max } \mathrm{I}-\mathrm{Z}\right) \mathrm{W}=0$, where $\mathrm{W}$ is a non-zero vector (eigenvector), and $\lambda_{\max }$ is a scalar value (eigenvalue). The normalized eigenvector is normalization of priorities of matrix $\mathrm{Z}$ represented as $w_{i}=\mathrm{Ni} / \mathrm{T}_{\mathrm{N}}$, where $w_{i}>0, \quad \sum_{i=1}^{n} w_{i}=1$; where $w_{i}$ is local priorities (normalized priorities) of eigenvector $\left(w_{1}, w_{2} \ldots w_{n}\right), \mathrm{T}_{\mathrm{N}}$ is total sum of priorities.

(a) Next, we carry out a consistency check of the pair-wise matrix for all computed matrices across the application hierarchy. The consistency check is accomplished from the consistency ratio (C.R) and is represented in the Eq. (3) given below:

C.R = Consistency Index (C.I) / Random index (R.I); where is C.I $=\frac{\lambda_{\max }-n}{n-1}$

Hence, the pair-wise matrix $\mathrm{Z}$ is supposed to be consistent so long as C.R $>0.1$

Step 6: In this important step, all the weight priorities computed from several matrices in step 5 are pulled together in the form of an aggregation matrix. Final analysis is performed to quantify performance outcome based on customized derived equations of the considered application problem.

\section{B. Quantification of a two-stage performance assessment of flexible CNT biosensor using AHP}




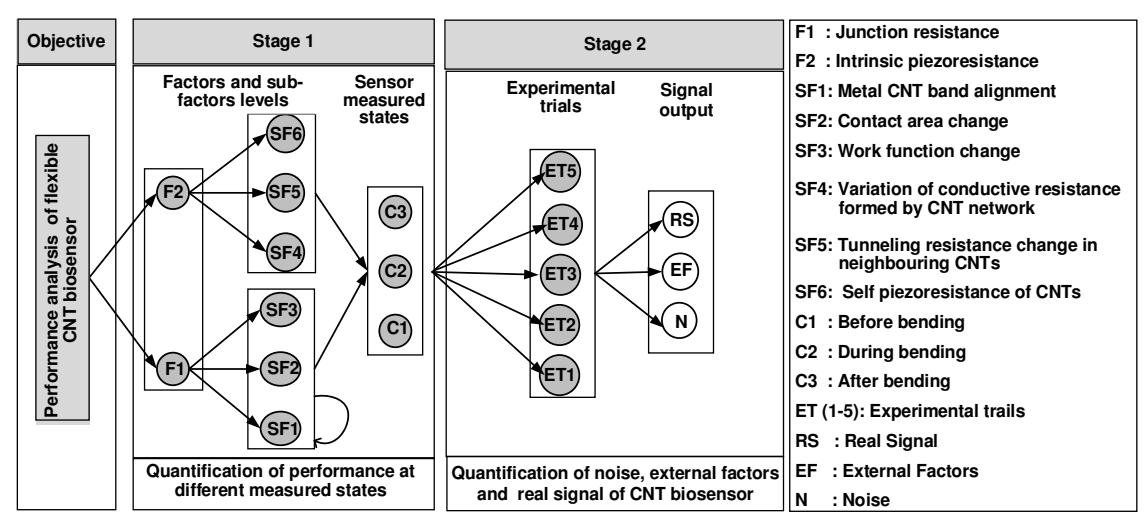

Fig. 6 Performance analysis of CNT biosensor using AHP- A two stage quantification assessment.

In this section, we briefly address the performance analysis of flexible CNT biosensor using AHP methodology with respect to the considered application discussed in the paper. Therefore, quantification of performance assessment is accomplished using a two-stage evaluation procedure (Figure 6). Stage 1 determines influencing factors at various measured states of the CNT biosensor, whereas Stage 2 determines various signal outcomes of flexible CNT biosensor during the sensing mode. This has been illustrated with our application of constructing a quantitative performance model by identifying various influencing factors. Thus, we have applied step-bystep AHP algorithm illustrated in Section IVA to quantify the performance of flexible CNT biosensor. This paper attempts to bridge the analytical performance model with experimental observations in a pragmatic manner. We have obtained the qualitative input for the various identified factor elements from several investigational trials by the following lead sources of data:

(a) obtained through experimental interpretations;

(b) via expert judgement observations;

(c) in combination via partially from (a) and (b) -i.e., from experimental data, and along with expert judgement to compensate missing/inconclusive/ambiguous data.

Therefore, we have performed extensive data analysis based on the availability of data from various sources that influence the final outcome to quantify flexible CNT biosensor performance for a precise estimate. In other words, aggregates various data observations through qualitative content breakdown analysis obtained from several repetitious experiments. Thus, Fig. 6 illustrates by defining the flexible CNT biosensor application's objective and constituting the factors at different levels of hierarchy (Steps 1 \& 2 of AHP procedure). Therefore, during the Stage 1 assessment, we have identified various factor elements influencing the performance during $\mathrm{C} 1, \mathrm{C} 2$, and $\mathrm{C} 3$ states of flexible CNT biosensor. Accordingly, modeled a three- level hierarchy of our considered application with factors (first level) and sub-factors (second level) affecting the performance of flexible CNT biosensor shown in Fig. 6. Whereas, in the Stage 2 assessment, we have quantified noise $(\mathrm{N})$ and other external factors (EF) contributing to the real signal (RS) of CNT biosensor reflecting its performance. Consequently, we have conducted 5 experimental trials (ET) with their relative comparisons. Accordingly, modeled a two-level hierarchy for each experimental trial ET (1-5) observations on the first level. The last level consists of various evaluating signal due to RS, EF, and N.

In brief, the performance assessment during these stages is executed analytically based on experimental observations with respect to:

TABLE 1

ASSESSMENT OF PAIRWISE COMPARISON MATRICES: STAGE 1 OF CNT BIOSENSOR PERFORMANCE. (a) COMPARISON MATRIX AT FACTOR LEVEL (b) COMPARISON MATRIX AT SUB-FACTOR LEVEL (c) COMPARISON MATRIX BETWEEN DIFFERENT OPERATING SENSING POSITIONS W.R.T SF1.

\begin{tabular}{|c|c|c|c|}
\hline & F1 & F2 & \multirow{2}{*}{$\begin{array}{c}\text { Normalized } \\
\text { priorities }\end{array}$} \\
\hline & \multicolumn{2}{|c|}{ Relative importance between F1, F2 } & \\
\hline F1 & 1 & 2 & $0.666(a)$ \\
\hline $\mathbf{F 2}$ & $1 / 2$ & 1 & $0.333(\mathrm{~b})$ \\
\hline \multicolumn{4}{|c|}{ Consistency ratio (C.R) $=0.00$} \\
\hline
\end{tabular}

(a)

\begin{tabular}{|l|c|c|c|c|c|c|}
\hline & SF1 & & SF2 & SF3 & Normalized & \\
\cline { 1 - 1 } & & \multicolumn{2}{c|}{$\begin{array}{l}\text { Relative importance } \\
\text { between SF1, SF2, SF3 }\end{array}$} & & \\
\hline SF1 & 1 & & 2 & 2 & $0.493\left(\mathrm{a}_{1}\right)$ & \\
\hline SF2 & $1 / 2$ & & 1 & 2 & $0.310\left(\mathrm{a}_{2}\right)$ & \\
\hline SF3 & $1 / 2$ & \multicolumn{7}{|c|}{ Consistency ratio (C.R) $=0.051$} & \\
\hline \multicolumn{7}{|c|}{} \\
\hline
\end{tabular}

(b)

\begin{tabular}{|c|c|c|c|c|}
\hline SF1 & $\mathrm{C1}$ & $\mathrm{C2}$ & $\mathbf{C 3}$ & \multirow{2}{*}{$\begin{array}{c}\text { Normalized } \\
\text { priorities }\end{array}$} \\
\hline & \multicolumn{3}{|c|}{ Likeliness of bending stress of SF1 } & \\
\hline C1 & 1 & $1 / 6$ & 1 & $0.129\left(\mathrm{a}_{11}\right)$ \\
\hline $\mathrm{C2}$ & 6 & 1 & 5 & $0.732\left(a_{12}\right)$ \\
\hline C3 & 1 & $1 / 5$ & 1 & $0.137\left(\mathrm{a}_{13}\right)$ \\
\hline \multicolumn{5}{|c|}{ Consistency ratio $(\mathrm{C} . \mathrm{R})=0.003$} \\
\hline
\end{tabular}


TABLE 2

ASSESSMENT OF PAIRWISE COMPARISON MATRICES: STAGE 2 OF CNT BIOSENSOR SIGNAL EVALUATION. (a) COMPARISON MATRIX AT ET (1-5). (B) COMPARISON MATRIX BETWEEN DIFFERENT OPERATING SENSING POSITIONS W.R.T RS

\begin{tabular}{|c|c|c|c|c|c|c|}
\hline & ET1 & ET2 & ET3 & ET4 & ET5 & $\begin{array}{c}\text { Normalized } \\
\text { priorities }\end{array}$ \\
\hline & \multicolumn{2}{|c|}{ Relative importance between ET1, ET2, ET3, ET4, ET5 } & $0.248\left(\mathrm{e}_{1}\right)$ \\
\hline ET1 & 1 & 1 & 1 & 2 & 2 & $0.248\left(\mathrm{e}_{2}\right)$ \\
\hline ET2 & 1 & 1 & 1 & 2 & 2 & $0.222\left(\mathrm{e}_{3}\right)$ \\
\hline ET3 & 1 & 1 & 1 & 2 & 1 & $0.149\left(\mathrm{e}_{4}\right)$ \\
\hline ET4 & $1 / 2$ & $1 / 2$ & $1 / 2$ & 1 & 2 & $0.131\left(\mathrm{e}_{5}\right)$ \\
\hline ET5 & $1 / 2$ & $1 / 2$ & 1 & $1 / 2$ & 1 & \\
\hline \multicolumn{7}{|c|}{ Consistency ratio (C.R) $=0.03$} \\
\hline
\end{tabular}

(a)

\begin{tabular}{|c|c|c|c|c|}
\hline & $\mathbf{R S}$ & EF & $\mathbf{N}$ & \multirow{2}{*}{$\begin{array}{c}\text { Normalized } \\
\text { priorities }\end{array}$} \\
\hline & \multicolumn{3}{|c|}{$\begin{array}{c}\text { Likeliness of signal } \\
\text { during RS }\end{array}$} & \\
\hline RS & 1 & 8 & 7 & $0.789\left(\mathrm{e}_{11}\right)$ \\
\hline $\mathbf{E F}$ & $1 / 8$ & 1 & 1 & $0.103\left(\mathrm{e}_{12}\right)$ \\
\hline $\mathbf{N}$ & $1 / 7$ & 1 & 1 & $0.107\left(\mathrm{e}_{13}\right)$ \\
\hline \multicolumn{5}{|c|}{ Consistency ratio $(\mathrm{C} . \mathrm{R})=0.001$} \\
\hline
\end{tabular}

TABLE 3

AGGREGATED MATRIX: PERFORMANCE OF CNT BIOSENSOR (STAGE 1 ASSESSMENT)

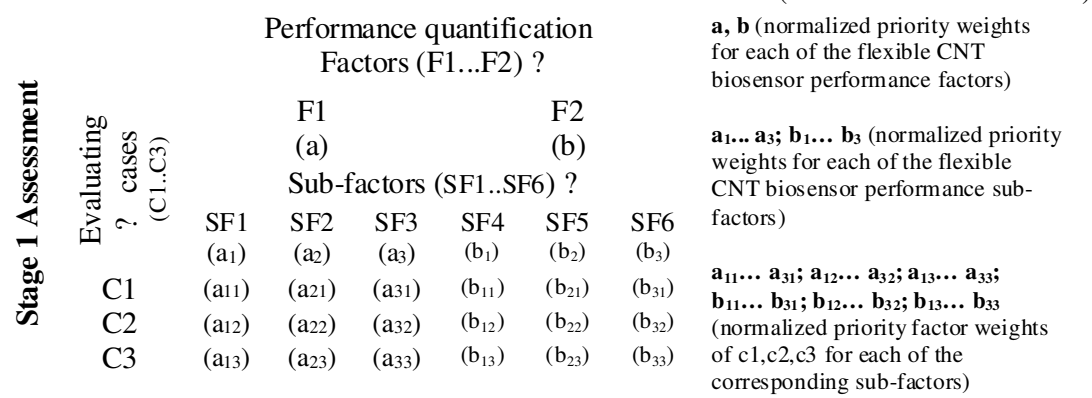

- CNT biosensor performance at C1, C2, and C3 (stage 1)

TABLE 4

- quantification of measured signal due to (i) RS (ii) $\mathrm{N}$ and (iii) EF (stage 2).

Based on the hierarchy structure we construct pair-wise comparisons matrices across the hierarchy of flexible CNT biosensor application among the (i) factors; (ii) subfactors;(iii) experimental trials and (iv) possible options and outcome; Subsequently, we compare relative importance between factor elements across the hierarchy influencing the CNT performance based on the experimental data, hands-on (expert judgement) and experimental set-up observations through the 9-point scale ratings proposed by Saaty [20] and computed normalized eigenvector (normalized priorities) associated with its principal eigenvalue (largest eigenvalue). The formation and computations of the matrix illustrate steps 3, 4 and 5 of the AHP procedure. Stage 1 model analysis is summarized in Table 1, which presents a few examples of assessed pair-wise comparison across the three levels of hierarchy. Table 1 (a) demonstrates pair-wise comparison between the factors (F1 and F2) at first level; Table 1(b) shows comparisons between sub-factors (SF1, SF2 \& SF3) at second level; finally, Table 1 (c) illustrates comparisons between C1, C2, \& C3 w.r.t SF1. Eventually, based on the AHP model (stage 1), we formulate and compute several pairwise matrices representing:

- between factor F1, F2 (formation of one matrix)

- between sub-factors SF1, SF2, \& SF3; and SF4, SF5, \& SF6 (formations of 2 matrices)

- likeliness of $\mathrm{C} 1, \mathrm{C} 2, \& \mathrm{C} 3$ in respect to each of the subfactors SF1 to SF6 (formulations of 6 matrices)

Stage 2 model analysis is summarized in Table 2, which presents a few examples of assessed a pair-wise comparison across the two levels of hierarchy. Table 2 (a) demonstrates pair-wise comparison between experimental trials (ET1, ET2, ET3, ET4, ET5) at the first level; while, Table 2 (b) illustrates

comparisons between RS, EF and $\mathrm{N}$ w.r.t EF1. Therefore, based on stage 2 of the AHP model, we formulate and compute the following pair-wise matrices representing:

- between ET1 to ET5 (formation of one matrix)

- likeliness of $\mathrm{RS}, \mathrm{EF}$, and $\mathrm{N}$ in respect to each of experimental trials ET1 to ET5 (formations of 5 matrices)

Finally, all the weight priorities computed from several matrices in step 5 are pulled together via. aggregation of weights in the form aggregation matrix.

We accomplish the concluding analysis from these aggregations of normalized priorities weights to quantify the overall performance of CNT biosensor outcome at the objective level. The calculations of final results depend on customized derived equations based on a number of hierarchy levels; a number of factor elements (factors, sub-factors) and evaluating options of the considered application problem. Table 3 presents an aggregation matrix for the performance of CNT biosensor (Stage 1), which pool up all normalized priorities computed as per the AHP modeling. Thus, the derived Eq. (4) quantifies the performance during C1. Finally, Eq. (5) provide a generalized derived equation that quantifies the performance of $\mathrm{CNT}$ biosensor for $\mathrm{C} 1, \mathrm{C} 2$, and $\mathrm{C} 3$. The 
TABLE 5

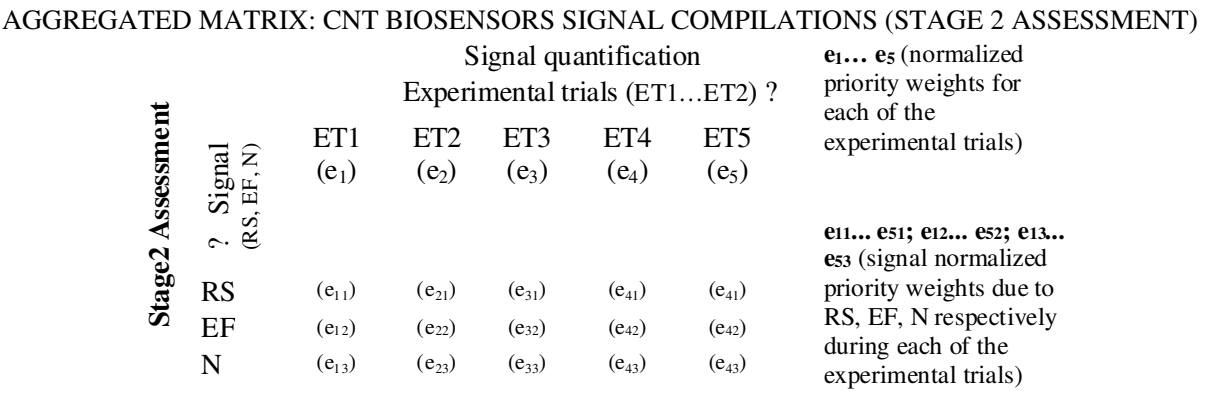

TABLE 6 EVALUATION OF SIGNAL QUANTIFICATION

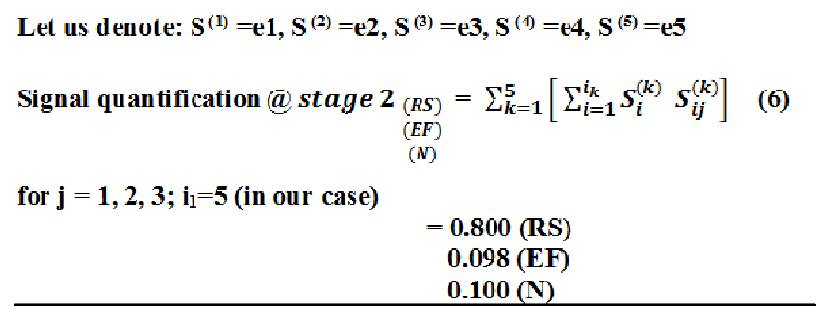

magnitude of the final calculated number indicates the degree to which the performance is affected during different operating sensing positions. We found that the flexible CNT biosensor has high priority value during $C 2$ (bending), with the highest value of $\sim \mathbf{0 . 7 3 2 8}$ when compared with other measured states $-C l$ (with a value of $\sim 0.1149$ ) and $C 3$ (with a value of 0.1522 ) (as summarized in Table 4). Thus, the obtained performance results from Stage 1 assessment indicate the highest impact of various influencing factors during bending. Further, Table 5, presents aggregation matrix of CNT signal due to RS, N, and EF (stage 2), resulting from an accumulation of normalized priorities as per the AHP model. Therefore, Eq. (6) quantifies CNT biosensor signals due to RS, N, and EF during sensing. Consequently, based on derived Eq. (6), we found $\boldsymbol{R S}$ with a value of $\sim \mathbf{0 . 8 0 0} ; \boldsymbol{N}$ signal with a value of $\sim 0.1008$ and signal due to $\boldsymbol{E F}$ with a value of $\sim \mathbf{0 . 0 9 8}$ as summarized in Table 6. Stage 2 analysis therefore shows that the normalized signal from the CNT sensor is comprised of noise $(\sim 9.8 \%)$, other external factors $(\sim 10.1 \%)$ and real signal ( $\sim 80 \%)$. Therefore, our AHP quantitative performance analysis at Stage $1 \& 2$ indicates a significant degree of impact in terms of numerical results over qualitative and traditional statistical methods.

\section{CONCLUSION}

The CNT network-based DNA sensors were fabricated on flexible PET by combining ink-jet printing and drop casting method. We have further carried out quantitative performance analysis of flexible CNT biosensors using a two-stage evaluation procedure. At Stage 1, influencing factors at various measured states of the CNT biosensor were identified and quantified, whereas, at Stage 2, various signal outcomes of flexible CNT biosensor during the sensing mode were determined. The quantification of sensor performance will emphases on the reliability of device to device signal variation through experimental trails. In our case, we have conducted multiple experimental trials reflecting the reproducibility of sensor functionality for our defined sets of objective requirements. We believe that by interpreting the overall outcome through the quantified results, we can improve the performance of flexible CNT biosensors.

\section{ACKNOWLEDGMENT}

The authors would also like to acknowledge Kenneth C. Cadien and Anastasia Elias for useful discussions and comments to improve the manuscript.

\section{REFERENCES}

[1]C. Yeom, K. Chen, D. Kiriya, Z. Yu, G. Cho, and A. Javey, "Large-Area Compliant Tactile Sensors Using Printed Carbon Nanotube Active-Matrix Backplanes," Adv. Mater., vol. 27, no. 9, pp. 1561-1566, 2015.

[2]T. Takahashi, Z. Yu, K. Chen, D. Kiriya, C. Wang, K. Takei, H. Shiraki, T. Chen, and A. Javey, "Carbon Nanotube Active-Matrix Backplanes for Mechanically Flexible Visible Light and X - ray Imagers," Nano Lett., vol. 13, pp. 5425-5430, 2013.

[3]P. H. Lau, K. Takei, C. Wang, Y. Ju, J. Kim, Z. Yu, T. Takahashi, G. Cho, and A. Javey, "Fully Printed, High Performance Carbon Nanotube Thin-Film Transistors on Flexible Substrates," Nano Lett., vol. 13, p. 3864-3869, 2013.

[4]H. Ota, K. Chen, Y. Lin, D. Kiriya, H. Shiraki, Z. Yu, T. Ha, and A. Javey, "Highly deformable liquid-state heterojunction sensors," Nat. Commun., vol. 5, pp. 1-9, 2014.

[5]W. Gao, H. Y. Y. Nyein, Z. Shahpar, H. M. Fahad, K. Chen, S. Emaminejad, Y. Gao, L. Tai, H. Ota, E. Wu, J. Bullock, Y. Zeng, D. Lien, and A. Javey, "Wearable Microsensor Array for Multiplexed Heavy Metal Monitoring of Body Fluids," ACS Sensors, 2016.

[6]H. Yin, Y. Nyein, W. Gao, Z. Shahpar, S. Emaminejad, S. Challa, K. Chen, H. M. Fahad, L. Tai, H. Ota, R. W. Davis, and A. Javey, "A Wearable Electrochemical Platform for Noninvasive Simultaneous Monitoring of $\mathrm{Ca} 2+$ and pH," ACS Nano, vol. 10, pp. 7216-7224, 2016.

[7] K. Chen, W. Gao, S. Emaminejad, D. Kiriya, H. Ota, H. Yin, Y. Nyein, K. Takei, and A. Javey, "Printed Carbon Nanotube Electronics and Sensor Systems," Adv. Mater., vol. 28, pp. 4397-4414, 2016.

[8] S. Sivashankar, C. Sapsanis, U. Buttner, and K. N. Salama, "Flexible lowcost cardiovascular risk marker biosensor for point-of-care applications," Electron. Lett., vol. 51, no. 22, pp. 1746-1748, 2015.

[9]M. C. McAlpine, H. Ahmad, D. Wang, and J. R. Heath, "Highly ordered nanowire arrays on plastic substrates for ultrasensitive flexible chemical sensors.," Nat. Mater., vol. 6, no. 5, pp. 379-84, May 2007.

[10] O. Kanoun, C. Müller, A. Benchirouf, A. Sanli, T. Dinh, A. Al-Hamry, L. Bu, C. Gerlach, and A. Bouhamed, "Flexible Carbon Nanotube Films for High Performance Strain Sensors," Sensors, vol. 14, no. 6, pp. 10042-10071, 2014.

[11] Alamusi, N. Hu, H. Fukunaga, S. Atobe, Y. Liu, and J. Li, "Piezoresistive strain sensors made from carbon nanotubes based polymer nanocomposites," Sensors, vol. 11, no. 11, pp. 10691-10723, 2011.

[12] S. M. Aqeel, Z. Huang, J. Walton, C. Baker, D. Falkner, Z. Liu, and Z. Wang, "Polyvinylidene fluoride (PVDF)/polyacrylonitrile (PAN)/carbon nanotube nanocomposites for energy storage and conversion," Adv. Compos. Hybrid Mater., 2017.

[13] M. Zhao, L. Meng, L. Ma, L. Ma, X. Yang, Y. Huang, J. E. Ryu, A. Shankar, T. Li, C. Yan, and Z. Guo, "Layer-by-layer grafting CNTs onto carbon fibers surface for enhancing the interfacial properties of epoxy resin composites," Compos. Sci. Technol., vol. 154, pp. 28-36, 2017.

[14] X. Wang, X. Liu, H. Yuan, H. Liu, C. Liu, T. Li, C. Yan, X. Yan, C. 
Shen, and Z. Guo, "Non-covalently functionalized graphene strengthened poly(vinyl alcohol)," Mater. Des., vol. 139, pp. 372-379, 2018.

[15] W. Gao, S. Emaminejad, H. Yin, Y. Nyein, S. Challa, K. Chen, A. Peck, H. M. Fahad, H. Ota, H. Shiraki, D. Kiriya, D. Lien, and G. A. Brooks, "Fully integrated wearable sensor arrays for multiplexed in situ perspiration analysis," Nature, vol. 529, no. 7587, pp. 509-514, 2016.

[16] G. Gruner, "Carbon nanotube transistors for biosensing applications," Anal Bioanal Chem, vol. 384, pp. 322-335, 2006.

[17] S. M. Aqeel, Z. Wang, L. Than, G. Sreenivasulu, and X. Zeng, "Poly(vinylidene fluoride)/poly(acrylonitrile) - based superior hydrophobic piezoelectric solid derived by aligned carbon nanotubes in electrospinning: fabrication, phase conversion and surface energy," RSC Adv., vol. 5, no. 93, pp. 76383-76391, 2015.

[18] S. Thomas L, "A scaling method for priorities in hierarchical structures," J. Math. Psychol., vol. 15, no. 3, pp. 234-281, 1977.

[19] Y. Wind and T. L. Saaty, "Marketing Applications of the Analytic Hierarchy Process," Manage. Sci., vol. 26, no. 7, pp. 641-658, 1980.

[20] T. L. Saaty, "Decision-making with the AHP: Why is the principal eigenvector necessary," vol. 145, pp. 85-91, 2003.

[21] M. A. Mustafa and J. F. Al-Bahar, "Project risk assessment using the analytic hierarchy process," Eng. Manag. IEEE Trans., vol. 38, no. 1, pp. 4652, 1991.

[22] J. Cancela, G. Fico, and M. T. Arredondo Waldmeyer, "Using the Analytic Hierarchy Process (AHP) to understand the most important factors to design and evaluate a telehealth system for Parkinson's disease," BMC Med. Inform. Decis. Mak., vol. 15, no. 3, pp. 1-11, 2015.

[23] W. C. Lee, F. H. Hung, K. F. Tsang, H. C. Tung, W. H. Lau, V. Rakocevic, and L. L. Lai, "A speedy cardiovascular diseases classifier using multiple criteria decision analysis," Sensors (Switzerland), vol. 15, no. 1, pp. 1312-1320, 2015.

[24] K. Schmidt, I. Aumann, I. Hollander, K. Damm, and J. M. G. Von Der Schulenburg, "Applying the Analytic Hierarchy Process in healthcare research: A systematic literature review and evaluation of reporting," $B M C$ Med. Inform. Decis. Mak., vol. 15, no. 1, 2015.

[25] Z.-P. Jia, Z.-Q. Huang, and Y.-X. Zhang, "Performance Evaluation of Wireless Sensor Networks Based on Entropy Weight and AHP," vol. 44, no. Wcsn 2016, pp. 640-645, 2017.

[26] K. K. Mohan, K. Prashanthi, R. Hull, and C. D. Montemagno, "Risk Assessment of a Multiplexed Carbon Nanotube Network Biosensor," IEEE Sens. J., vol. 18, no. 11, pp. 4517-4528, 2018.

[27] Z. Shen and Q. Wang, "A novel health evaluation strategy for multifunctional self-validating sensors," Sensors, vol. 13, no. 1, pp. 587-610, 2013.

[28] A. Nag, B. Menzies, and S. C. Mukhopadhyay, "Performance analysis of flexible printed sensors for robotic arm applications," Sensors Actuators, A Phys., vol. 276, pp. 226-236, 2018.

[29] A. Maric, M. Kisic, G. Radosavljevic, N. Blaz, L. Zivanov, K. Babkovic, and M. Damnjanovic, "Performance analysis of a flexible polyimide based device for displacement sensing," Facta Univ. - Ser. Electron. Energ., vol. 28, no. 2, pp. 287-296, 2015.

[30] D. Z. Vasiljevic, A. Mansouri, L. Anzi, R. Sordan, and G. M. Stojanovic, "Performance Analysis of Flexible Ink-Jet Printed Humidity Sensors Based on Graphene Oxide," IEEE Sens. J., vol. 18, no. 11, pp. 43784383, 2018.

[31] A. Star, E. Tu, J. Niemann, J. P. Gabriel, C. S. Joiner, and C. Valcke, "Label-free detection of DNA hybridization using carbon nanotube network field-effect transistors," Proc. Natl. Acad. Sci., vol. 103, no. 4, pp. 921-926, 2006.

[32] J. Wu, R. Wang, H. Yu, G. Li, K. Xu, N. C. Tien, R. C. Roberts, and D. $\mathrm{Li}$, "Inkjet-printed microelectrodes on PDMS as biosensors for functionalized microfluidic systems," Lab Chip, vol. 15, no. 3, pp. 690-695, 2015.

[33] C. M. Silveira, T. Monteiro, and M. G. Almeida, "Biosensing with Paper-Based miniaturized printed electrodes-A modern trend," Biosensors, vol. 6, no. 4, pp. 1-17, 2016.

[34] L. F. C. Pereira and M. S. Ferreira, "Electronic transport on carbon nanotube networks: A multiscale computational approach," Nano Commun. Netw., vol. 2, no. 1, pp. 25-38, 2011.

[35] R. A. Bell, Conduction in Carbon Nanotube Networks: Large-Scale Theoretical Simulations. Springer International Publishing, 2015.

[36] S. Heinze, J. Tersoff, R. Martel, V. Derycke, J. Appenzeller, and P. Avouris, "Carbon Nanotubes as Schottky Barrier Transistors," Phys. Rev. Lett., vol. 89, no. 10, pp. 2-5, 2002.

[37] T. Yamada, "Modeling of carbon nanotube Schottky barrier modulation under oxidizing conditions," Phys. Rev. B - Condens. Matter Mater. Phys., vol. 69 , no. 12 , pp. 2-9, 2004.
[38] X. Tang, S. Bansaruntip, N. Nakayama, and E. Yenilmez, "Carbon Nanotube DNA Sensor and Sensing Mechanism," Nano Lett., vol. 6, no. 8, pp. 1632-1636, 2006.

[39] P. Bondavalli, P. Legagneux, and D. Pribat, "Carbon nanotubes based transistors as gas sensors: State of the art and critical review," Sensors Actuators, B Chem., vol. 140, no. 1, pp. 304-318, 2009.

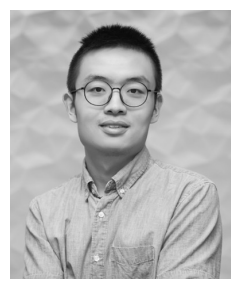

Liang Cai completed his bachelor degree in Electrical Engineering Co-op program at the University of Alberta (U of A, 2020). He joined the research team of Dr. Prashanthi Kovur in 2017 and worked as a research assistant for 8 months.

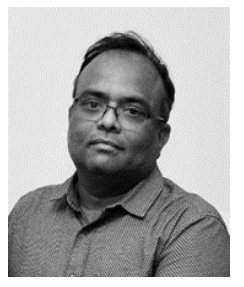

Dr. K. Krishna Mohan received his $\mathrm{PhD}$ in Electrical Engineering from Indian Institute of Technology (IIT) Bombay in 2011. He has worked as a consultant on uncertainty analysis, quantification and risk assessment of nanobiosensors in the Department of Chemical \& Materials Engineering at the University of Alberta ( $U$ of A). He also worked as a postdoctoral fellow in the department of Electrical \& Computer Engineering at $U$ of $A$ and was actively involved in the risk management and information technology for agriculture wellness and disease out-breaks. Before joining $\mathrm{U}$ of $\mathrm{A}$, he worked in the field of software reliability for mission critical software systems. He received the best thesis award from Engineering Sciences from IIT-Bombay in 2011 and also recipient of "2009 IEEE Reliability Society Award".

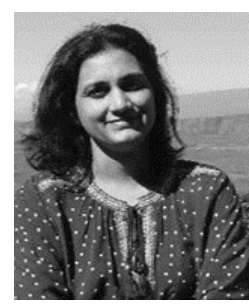

Dr. Prashanthi Kovur worked as a Research Associate in the Department of Chemical and Materials Engineering at University of Alberta. She received her Ph.D. degree from the Department of Electrical Engineering at Indian Institute of Technology Bombay (IITB) in 2010. Her current research focuses on micro/nanofabrication of devices using 1D, 2D functional nanomaterials and development of multiplexed nanobiosensors on flexible \& silicon platforms for agriculture and biological applications.

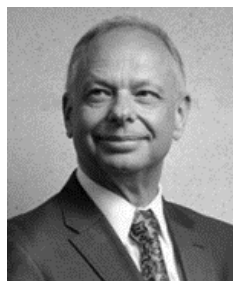

Dr. Carlo D. Montemagno received the B.Sc. degree in agricultural and biological engineering from Cornell University in 1980, the M.Sc. degree in petroleum and natural gas engineering from Penn State University in 1990, and the $\mathrm{Ph} . \mathrm{D}$. degree in civil engineering and geological sciences from Notre Dame University in 1995. He has served as a Chancellor and a Professor of Engineering with Southern Illinois University (SIU) Carbondale. He is an internationally recognized expert in nanotechnology and biomedical engineering, with a focus on linking multiple disciplines to solve problems in areas of health, energy, and the environment. Prior to his appointment at SIU, he founded the interdisciplinary Ingenuity Lab based at the University of Alberta in Canada. In addition to leading the lab, which connects organizations and researchers from across the Province of Alberta, he served as Director of the biomaterials program for the Canadian Research Council's National Institute for Nanotechnology as well as Research Chair in intelligent nanosystems for the Canadian National Research Council. 